\title{
A family of low-cost transfer orbits for rendezvous-type missions to NEAs
}

\author{
Ştefan Berinde \\ Babeş-Bolyai University, Cluj-Napoca, Romania \\ email: sberinde@math.ubbcluj.ro
}

\begin{abstract}
We develop a family of transfer orbits suitable for the peculiarity of NEAs orbits. They involve one or more carefully planned deep-space maneuvers and one or more close encounters with the Earth. Basically, it is a gravity assist technique, but only Earth is used as the driving planet. In this manner, the orbit of the spacecraft stays near the Earth, as the orbits of NEAs. The idea is to obtain a large relative velocity in respect to the Earth, using these impulsive maneuvers. Next, successive rotations of this relative velocity vector due to resonant encounters with the Earth will, finally, shape the orbit of the spacecraft, in order to match the inclination and the orientation of apsidal line of the asteroid's orbit.

With a velocity budget less than $9 \mathrm{~km} / \mathrm{s}$ most of NEAs orbits are reachable, even highly inclined ones, or orbits with relatively large nodal distances. An example is given: a rendezvous mission to asteroid (1036) Ganymed.
\end{abstract}

Keywords. space missions; rendezvous; resonant returns

\section{Introduction}

The population of discovered near-Earth asteroids (NEAs) is continuously growing. They mimic various (and mostly unknown) physical characteristics (internal structure, rotation, companions, etc.) which qualify them for in situ exploration. From the dynamical point of view, many of them have orbits allowing close encounters with the Earth in the near or distant future. Mitigation of an impact hazard might require in situ exploration of these objects. Among the types of possible interplanetary missions: rendezvous, nodal flyby or resonant flyby missions (Perozzi et al. 2001), the first one offers the greatest scientific return, since the spacecraft will stay longer near the object to study or interact with it. Rendezvous missions require a transfer orbit in such a way that the spacecraft will end on the same orbit as the target asteroid. This alteration of the orbit is usually very expensive in terms of velocity budget. Even if NEAs are "near" the Earth, many of them are not easily reachable on rendezvous missions.

In this paper we develop a family of transfer orbits for rendezvous missions, equally applicable to most of the NEAs, with a total velocity budget at a reasonable level $(<9 \mathrm{~km} / \mathrm{s})$. The orbits involve deep-space maneuvers (DSMs), and one or more close encounters with the Earth. Being rather complex, the major side effect is the large transfer time, sometimes above 10 years. We use the piecewise two-body approximation for this approach. Next section will summarize this theory. In section 3 we introduce the concept of true intersection orbits. In section 4 we analyze the velocity requirements to accomplish a rendezvous-type mission to NEAs using simple transfers (two-impulse transfers). In section 5 we introduce a technique to increase the velocity of a spacecraft in respect to the Earth using deep-space maneuvers and in section 6 we show how to build highly inclined orbits using resonant encounters with our planet. In section 7 we give a complete example of how the theory works and, finally, we draw some conclusions. 


\section{Piecewise two-body approximation}

Piecewise two-body approximation is basically a two "two-body" problem approach for the motion of an infinitesimal body in interplanetary space (Carusi et al. 1990). It can be viewed as a simplified version of patched-conics technique used in the field of Astrodynamics (Battin 1987). We recall some of its principles: Earth (the perturbing planet in this case) moves in circular orbit around the Sun, the spacecraft (the infinitesimal body) has a geocentric hyperbolic orbit near the planet and an elliptic heliocentric orbit in interplanetary space. The geocentric phase is in fact an instantaneous event (close encounter), intended to change the orientation of the unperturbed geocentric relative velocity vector of the spacecraft (Figure 1a).

We adopt the following units for distance and time, such that the radius of the Earth's orbit equals 1 and its heliocentric velocity is also 1 . It follows that heliocentric gravitational parameter is $\mu_{\odot}=1$ and Earth's orbital period is $T_{\oplus}=2 \pi$.

The heliocentric orbit is given as a set of well known keplerian elements $(a, e, i)$. The other two ones $\omega$ and $\Omega$ are not independent variables (since the orbits must intersect each other at a known location). The geocentric orbit is fully described by the unperturbed geocentric relative velocity (in short, relative velocity) $u$, which is an invariant of the encounter, and the orientation of this velocity vector, given by two angles $(\theta, \phi)$. We do not reproduce here the formulas relating these variables, just say that the heliocentric orbit can be propagated analytically through a close encounter, as follows

$$
(a, e, i) \longrightarrow(u, \theta, \phi) \longrightarrow\left(u, \theta^{\prime}, \phi^{\prime}\right) \longrightarrow\left(a^{\prime}, e^{\prime}, i^{\prime}\right)
$$

The link between the two sets of angles $(\theta, \phi)$ and $\left(\theta^{\prime}, \phi^{\prime}\right)$ involves two additional angles: the deflection angle $\gamma$ and the inclination of geocentric orbit $\psi$ in respect to a given reference plane. The deflection angle gives a measure of how much the encounter affects the orbit of the spacecraft, and its expression is

$$
\sin \frac{\gamma}{2}=\left[1+\frac{r_{\min }}{r_{L E O}}\left(\frac{u}{v_{L E O}}\right)^{2}\right]^{-1}, \quad r_{\min } \geqslant r_{L E O}
$$

It depends on the minimum distance of the encounter $r_{m i n}$, but also on the physical properties of the planet, which are embedded in the values of the circular velocity in low-Earth orbit $(\mathrm{LEO}), v_{L E O}=0.26(7.8 \mathrm{~km} / \mathrm{s})$, and in the radius of LEO orbit $r_{L E O}$. We set this value to be at an altitude of $200 \mathrm{~km}$ above the Earth's surface. This is also the minimum allowed altitude for the encounter. So, the maximum value for the deflection angle, $\gamma_{\max }$, is obtained for $r_{\min }=r_{L E O}$.

\section{True intersection orbits}

It is useful for our approach to introduce the following concept. We define the true intersection orbit related with the orbit of a NEA, that orbit which geometrically intersects the Earth's one and is obtained from the original orbit using a minimum single-impulse transfer (Figure 1b).

The velocity increment $d v_{0}$ required to change an orbit to a true intersection one can be computed numerically. However, for the sake of simplicity we use the following analytical approximation. We consider that the velocity $d v_{0}$ is applied along the heliocentric velocity vector at predefined locations on original orbit (aphelion, perihelion or a nodal point). In this manner, the nodal distances changes till one of them equals 1 (true intersection). The orbital inclination is not altered nor the longitude of ascending node.

It's easy to compute explicit formulas for this velocity increment applied at aphelion $d v_{Q}$, perihelion $d v_{q}$ and at the opposite orbital node $d v_{n}$. Since there are two nodal 

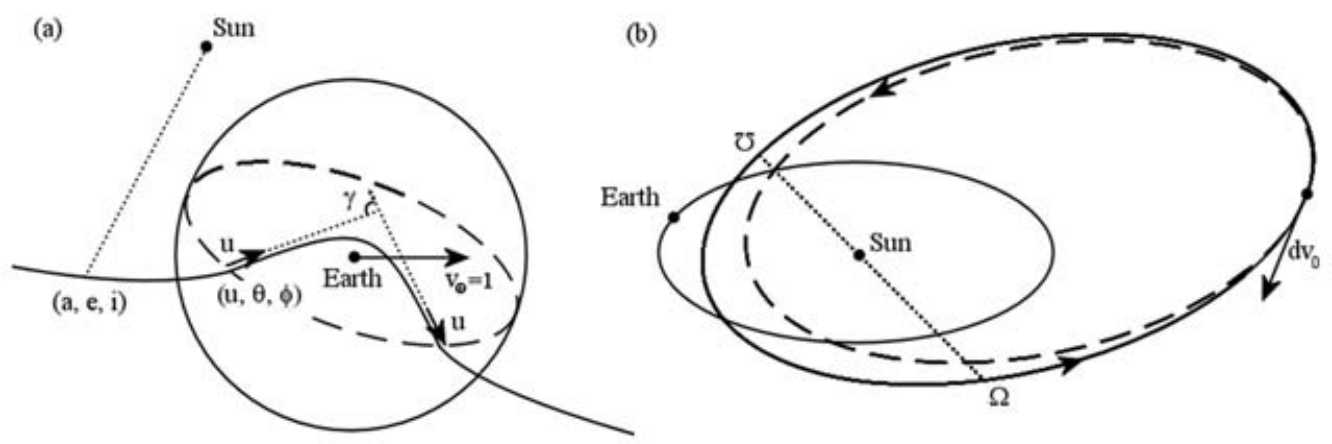

Figure 1. (a) Geometry of piecewise two-body approximation; (b) The true intersection orbit (dotted) of an original non-intersecting one (solid).

distances aimed to be changed, the minimum velocity increment is computed as $d v_{0}=$ $\min \left\{d v_{Q}^{1}, d v_{Q}^{2}, d v_{q}^{1}, d v_{q}^{2}, d v_{n}^{1}, d v_{n}^{2}\right\}$, where superscript selects the nodal distance involved in computation. Using a simple plot we conclude that most of the discovered NEAs require $d v_{0}<0.1(3 \mathrm{~km} / \mathrm{s})$.

If $\left(Q_{0}, q_{0}\right)$ denotes the aphelion and perihelion distances of the true intersection orbit, the geocentric relative velocity at the intersection point is computed from

$$
u_{0}^{2}=3-\frac{2}{Q_{0}+q_{0}}-\sqrt{\frac{8 Q_{0} q_{0}}{Q_{0}+q_{0}}} \cos i
$$

In this manner, each orbit of a NEA has an associated geocentric relative velocity $u_{0}$.

\section{Velocity requirements for rendezvous-type missions}

The most expensive task in a rendezvous mission is to match the orbital inclination of the target asteroid. Also, the complexity of the mission depends on how distant the orbit is, in respect to the Earth's one. For orbits not very close to the ecliptic, this aspect is quantified by the closest nodal distance of the asteroid's orbit. In Figure 2a we depict the distribution of discovered NEAs function of these two orbital parameters. There is an apparent clustering of orbits having an orbital node near the Earth's orbit and low orbital inclination, an effect of observational bias. Anyway, many NEAs have "wild" orbits, even if, by definition, they are "near" the Earth.

It becomes clear that a rendezvous mission to such bodies involves a high velocity budget. We will give a measure of this budget below, using a simple (and naive) tranfer scenario: two-impulse orbital transfer. First impulse $d v_{\text {node }}$ is performed in Earth's orbit, in order to obtain a Hohmann transfer to one of the orbital nodes of the NEA's orbit (most favourable one). Its expression is

$$
d v_{\text {node }}=\sqrt{\frac{2 d}{(1+d)}}-1
$$

where $d$ is the nodal distance of the selected node. From here, a second impulse, given by

$$
u_{\text {node }}^{2}=\frac{2(2+d)}{d(1+d)}-\frac{1}{a}-\frac{2}{d} \sqrt{\frac{2 a\left(1-e^{2}\right)}{d(1+d)}} \cos i
$$



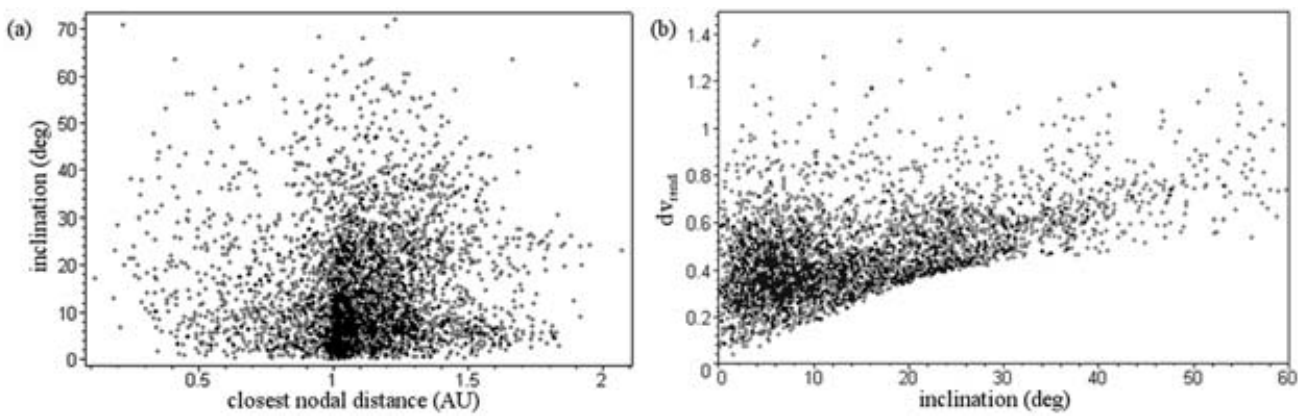

Figure 2. (a) Distribution of discovered NEAs in the plane of closest nodal distance (in respect to Earth's orbit) versus orbital inclination; (b) Velocity budget required to accomplish a rendezvous mission to each discovered NEA versus their orbital inclination (see text). Values on the vertical axis should be multiplied roughly by 30 to get the velocity expressed in $\mathrm{km} / \mathrm{s}$.

will put the spacecraft on the target orbit $(a, e, i)$. The total velocity budget is now $d v_{\text {rend }}=d v_{\text {node }}+u_{\text {node }}$ (Berinde 2005). This approach was actually used by Perozzi et al. (2001), in order to estimate the accessibility of the whole NEA population, but with additional assumptions corresponding to "best case" mission profiles. Figure $2 \mathrm{~b}$ depicts this velocity budget for each discovered NEA, function of the orbital inclination. Most of the NEAs require velocities much larger than $0.3(9 \mathrm{~km} / \mathrm{s})$, which we take it as the upper limit for a low-cost interplanetary mission.

\section{Increasing relative velocity with deep-space maneuvers}

In this section we describe a technique in which a spacecraft can gain high relative velocities in respect to the Earth, using several small velocity impulses along the transfer orbit (deep-space maneuvers). At first, we give the following portrait of an orbit intersecting the Earth's one and located entirely in the ecliptic plane. Let $u$ denotes the relative velocity at the intersection point and angle $\theta$ describes the orientation of this velocity vector against the heliocentric velocity vector of the Earth. The pair $(u, \theta)$ gives a complete description of the orbit. All other orbital quantities are computable, like semi-major axis $a$, orbital parameter $p$, aphelion distance $Q$ and true anomaly of the intersection point $f$. They are given bellow

$$
\begin{cases}\frac{1}{a}=1-2 u \cos \theta-u^{2}, & p=(1+u \cos \theta)^{2} \\ Q=a\left(1+\sqrt{1-\frac{p}{a}}\right), & \cos f=\frac{p-1}{1-p / Q}\end{cases}
$$

As in Figure 3a, let the orbit of a spacecraft, with parameters $\left(u_{1}, \theta_{1}\right)$, have an intersection point A with the Earth's orbit. At the aphelion point B a small velocity impulse $d v$ is applied in order to reduce the heliocentric velocity by this amount. Other two intersection points with the Earth's orbit appear, C (post-perihelion) and C' (pre-perihelion). Let $\left(u_{2}, \theta_{2}\right)$ be the new parameters of the orbit computed at one of these intersection points. We obtain the following relations

$$
\left\{\begin{array}{l}
d v=\sqrt{u_{2}^{2}+y}-\sqrt{u_{1}^{2}+y}, \quad \text { where } y=Q^{2}+\frac{2}{Q}-3 \\
Q d v=u_{1} \cos \theta_{1}-u_{2} \cos \theta_{2}
\end{array}\right.
$$



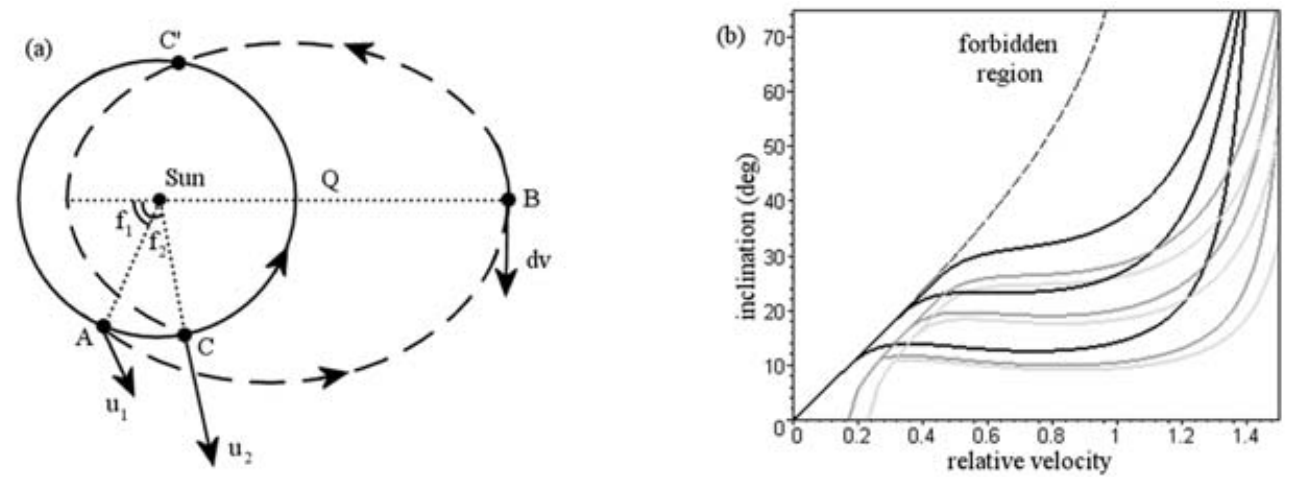

Figure 3. (a) Increasing relative velocity in respect to the Earth, using a deep-space maneuver; (b) Maximum value of the orbital inclination after one, two and three successive encounters with the Earth, on resonant orbits with semi-major axis $a_{1: 1}$ (black), $a_{2: 1}$ (dark gray) and $a_{3: 1}$ (light gray).

which enable us to express the parameters $\left(u_{2}, \theta_{2}\right)$ function of $\left(u_{1}, \theta_{1}\right)$ and $d v$. More than that, it can be shown that $u_{2}>u_{1}+d v$. The difference $d u=u_{2}-\left(u_{1}+d v\right)$ is significant, as can be seen later. It is an increasing function of $Q$ and $d v$, but decreasing in $u_{1}$.

We must ask for Earth and the spacecraft to depart simultaneously from point $\mathrm{A}$ and arrive also simultaneously in point $\mathrm{C}$ (or $\mathrm{C}^{\prime}$ ). We need to compute the travel times for each of these bodies. Let $\left(a_{1}, f_{1}, Q\right)$ be the set of elements derived from $\left(u_{1}, \theta_{1}\right)$, and $\left(a_{2}, f_{2}, Q\right)$ the set of elements derived from $\left(u_{2}, \theta_{2}\right)$ as shown in equations (5.1). If Earth passes $m$ times before the next encounter through the conjunction point with point $\mathrm{B}$, its travel time reads

$$
d t_{\oplus}=-f_{1}+f_{2}+2 \pi m
$$

If the spacecraft passes $n$ times before the next encounter through the aphelion point $\mathrm{B}$, its travel time reads

$$
d t=d t_{1}\left(a_{1}, f_{1}, Q\right)+d t_{2}\left(a_{2}, f_{2}, Q\right)+2 \pi(n-1) a_{2}{ }^{3 / 2},
$$

where $d t_{1}$ and $d t_{2}$ are computable, but their expressions are rather lengthy to be reproduced here (Battin 1987).

The motion of these two bodies is synchronized if $d t=d t_{\oplus}$. If the pair $\left(u_{1}, \theta_{1}\right)$ is given, there exist at most one value of the velocity impulse $d v$ for which the motion is synchronized. We name such a transfer orbit as of type " $\pm \frac{m}{n} \pm$ ". The sign "+" stands for a post-perihelion intersection point and "-" for a pre-perihelion intersection point. If there exist only one intersection point, we omit this sign. For example, a transfer orbit of type " $+\frac{2}{1}+"$ will start at point $\mathrm{A}$ and will end at point $\mathrm{C}$ as depicted in Figure 3a. A transfer orbit of type " $+\frac{1}{1}-"$ does not exist at all.

The process can be continued further. At the encounter point $\mathrm{C}$, the angle $\theta_{2}$ is altered due to a close encounter to a new value $\theta_{2}^{\prime}$, freely available in the interval $\left[\theta_{2}-\gamma_{\max }, \theta_{2}+\gamma_{\max }\right]$. The previous procedure is restarted with the new pair $\left(u_{2}, \theta_{2}^{\prime}\right)$. We obtain, eventually, a transfer orbit of type " $\pm \frac{m}{n} \pm \frac{m^{\prime}}{n^{\prime}} \pm$ ", and so on.

In practice, in order to maximize the efficiency of the process, we choose

$$
\left\{\begin{array}{l}
\theta_{1}=0 \\
\theta_{2}^{\prime}=\max \left\{0, \theta_{2}-\gamma_{\max }\right\} \\
\theta_{3}^{\prime}=\max \left\{0, \theta_{3}-\gamma_{\max }\right\} \\
\cdots
\end{array}\right.
$$


Table 1. Families of solutions used to increase the relative velocity in respect to the Earth. Values on the second and third column should be multiplied roughly by 30 to get the velocity expressed in $\mathrm{km} / \mathrm{s}$ (see text for details)

\begin{tabular}{lccc}
\hline transfer type & sum of all impulses & final relative velocity & travel time (years) \\
\hline $1 / 1+$ & $0.07-0.30$ & $0.14-0.62$ & $1.27-1.35$ \\
$2 / 1-$ & $0.17-0.30$ & $0.21-0.69$ & $1.93-1.70$ \\
$2 / 1+$ & $0.17-0.30$ & $0.17-0.69$ & $2.00-2.27$ \\
$1 / 1+2 / 1-$ & $0.09-0.29$ & $0.20-0.87$ & $2.91-3.21$ \\
$1 / 1+2 / 1+$ & $0.08-0.29$ & $0.17-0.93$ & $3.28-3.58$ \\
$1 / 1+2 / 1-2 / 1+$ & $0.17-0.26$ & $0.62-0.93$ & $5.47-5.55$ \\
$1 / 1+2 / 1+2 / 1+$ & $0.09-0.26$ & $0.20-0.93$ & $5.33-5.68$ \\
$1 / 1+2 / 1-3 / 1-$ & $0.10-0.22$ & $0.29-0.91$ & $5.85-6.10$ \\
$1 / 1+2 / 1+3 / 1-$ & $0.10-0.24$ & $0.27-0.97$ & $6.02-6.29$ \\
$1 / 1+2 / 1-3 / 1+$ & $0.09-0.23$ & $0.24-1.00$ & $6.20-6.50$ \\
$1 / 1+2 / 1+3 / 1+$ & $0.09-0.23$ & $0.23-1.00$ & $6.35-6.70$ \\
\hline
\end{tabular}

These assumptions produce maximum values for the aphelion distances on each transfer orbit and a minimum value for $u_{1}$. In this way, we get a maximized value for the final relative velocity in respect to the Earth. For each type of transfer orbit and for a given value of the final relative velocity, the starting value of $u_{1}$ is unique (if exist). Table 1 summarizes all practical types of transfer orbits, together with the range of sum of all impulses performed, the range of final relative velocities and the range of travel times until the last encounter. We have three categories of solution families: one-encounter solutions, two-encounter solutions and three-encounter solutions. The last ones are more efficient, but they require larger travel times (see table). We conclude here that a final relative velocity of at most $1.0(30 \mathrm{~km} / \mathrm{s})$ can be reached using a total velocity budget less than $0.23(7 \mathrm{~km} / \mathrm{s})$. We have limited our search to a reasonable range of orbital elements of transfer orbits, as follows $0.3=q_{\min } \leqslant q \leqslant 1 \leqslant Q \leqslant Q_{\max }=4.0$ (AU).

\section{Increasing orbital inclination with resonant encounters}

After completing the procedure from previous section, the final relative velocity vector is still on the ecliptic plane. Now, using one or more encounters with the Earth, this vector can be rotated, in order to match the inclination of the target orbit and, also, the orientation of its apsidal line. This process is performed without additional energy consumption, provided that this relative velocity is large enough.

If a single encounter cannot perform the desired inclination change, the spacecraft must be set on a resonant orbit with the Earth for an additional encounter at the same intersection point. We consider only the following mean motion resonances 1:1, 2:1, 3:1 and corresponding semi-major axis $a_{1: 1}, a_{2: 1}, a_{3: 1}$. For each of these semi-major axis we depict on Figure $3 b$ the maximum value of the orbital inclination after one, two and three successive encounters with the Earth. Low-order resonant orbits are more efficient and have shorter travel times, but they are not always accesible from the initial orbit. Sometimes is possible to change a resonant semi-major axis into another resonant semimajor axis after an additional encounter, in order to increase the efficiency and reduce total travel time. For a given relative velocity $u$, the maximum possible orbital inclination is $i=\arcsin u$, also depicted in the figure (dotted curve). 


\section{Putting all together: A random example}

Let suppose we have the orbit of an NEA and we want to target it for a rendezvous mission. We do not take here into account the phasing requirements between the spacecraft and the asteroid. We will show only how to build a low-cost transfer orbit.

- Step 1. Consider the true intersection orbit related with the original orbit of the asteroid. We record the longitude $\Omega$ (or $\mho$ ) of its intersection point with the Earth's orbit, the relative velocity $u_{0}$ at this point, the semi-major axis $a_{0}$ and the orbital inclination $i$ (the same as the original orbit).

- Step 2. Compute the synchronized transfer orbits used to increase the relative velocity exactly at a value of $u_{0}$. There is at most one solution for each type of transfer orbit. We choose that one which offers a good balance between the sum of required velocity impulses and travel time. The last encounter with the Earth should occur at the same longitude as recorded in step 1. This restricts the launch opportunity once every year.

- Step 3. Since the final relative velocity is exactly $u_{0}$, a proper orientation of this velocity vector will put the spacecraft on the true intersection orbit computed in step 1 . We aim for an orbital inclination $i$ and a final semi-major axis $a_{0}$. One or more encounters with the Earth on resonant orbits might be required to perform these orbital changes.

- Step 4. From the true intersection orbit a final impulse $d v_{0}$ is required to get into the asteroid's orbit.

This is a general purpose technique, equally applicable to most of the NEAs orbits. Only orbits with very high relative velocities, $u_{0}>1.0(30 \mathrm{~km} / \mathrm{s})$, or orbits with very large aphelion distances are not suitable for this technique. Also, there is no point to apply this method for orbits with low relative velocities $u_{0}<0.14(4 \mathrm{~km} / \mathrm{s})$, since a low-cost two-impulse transfer orbit does exist in this case.

To show the generality of our method, we pick up a random NEA as an example. It is random from the point of view of its orbital characteristics, but not from the physical point of view. Because we choose the largest known NEA - asteroid (1036) Ganymed. Some of its orbital elements are summarized in table 2. For sure, it is not an easy target.

Table 2. Some orbital parameters of the asteroid (1036) Ganymed

\begin{tabular}{cccccccc}
\hline$a$ & $e$ & $Q$ & $q$ & $d_{1}$ & $d_{2}$ & $i$ & $u_{0}$ \\
\hline 2.67 & 0.53 & 4.09 & 1.24 & 1.40 & 2.90 & $26.7^{\circ}$ & 0.67 \\
\hline
\end{tabular}

Among the types of transfer orbits found as solutions, we consider the " $\frac{1}{1}+\frac{2}{1}+$ " one, which offers a good compromise between total velocity budget and completion time. We summarize all important quantities in table 3. The mission is completed in 11.4 years with a velocity budget of $7.3 \mathrm{~km} / \mathrm{s}$. A schematic view of orbits is given in Figure 4 .

\section{Conclusions}

In this paper we introduce a low-cost rendezvous technique applicable to most of the NEAs orbits. It involves one or more carefully planned deep-space maneuvers and one or more close encounters with the Earth. Basically, it is a gravity assist technique, but only Earth is used as the driving planet. In this manner, the orbit of the spacecraft stays near the Earth, as the orbits of NEAs. With velocity budgets less than $9 \mathrm{~km} / \mathrm{s}$ most of NEAs orbits are reachable, even highly inclined ones, or orbits with relatively large nodal distances. A random example is given: a rendezvous mission to asteroid (1036) Ganymed.

Besides of cost advantages offered by this technique, we should discuss here its limitations. One is the completion time, which may easily go beyond 10 years, depending 
Table 3. Quantities related with the transfer orbit found for the asteroid (1036) Ganymed. The mission is completed in 11.4 years with a velocity budget of $7.3 \mathrm{~km} / \mathrm{s}$.

\begin{tabular}{cccccc}
\hline Event & $\begin{array}{c}\text { minimum enc. } \\
\text { altitude }(\mathrm{km})\end{array}$ & $\begin{array}{c}\text { geocentric relative } \\
\text { velocity }\end{array}$ & $\begin{array}{c}\text { orbital } \\
\text { incl. }(\mathrm{deg})\end{array}$ & $\begin{array}{c}\text { sum of velocity } \\
\text { impulses }\end{array}$ & $\begin{array}{c}\text { travel time } \\
\text { (years) }\end{array}$ \\
\hline launch & - & $0.05(1.3 \mathrm{~km} / \mathrm{s})$ & 0 & $0.05(1.3 \mathrm{~km} / \mathrm{s})$ & 0 \\
enc. \#1 & 200 & $0.21(6.4 \mathrm{~km} / \mathrm{s})$ & 0 & $0.11(3.1 \mathrm{~km} / \mathrm{s})$ & 1.3 \\
enc. \#2 & 200 & $0.67(20.2 \mathrm{~km} / \mathrm{s})$ & 10.2 & $0.20(6.0 \mathrm{~km} / \mathrm{s})$ & 3.5 \\
enc. \#3 & 200 & $0.67(20.2 \mathrm{~km} / \mathrm{s})$ & 19.2 & $0.20(6.0 \mathrm{~km} / \mathrm{s})$ & 5.5 \\
enc. \#4 & 200 & $0.67(20.2 \mathrm{~km} / \mathrm{s})$ & 26.3 & $0.20(6.0 \mathrm{~km} / \mathrm{s})$ & 7.5 \\
enc. \#5 & 1300 & $0.67(20.2 \mathrm{~km} / \mathrm{s})$ & 26.7 & $0.20(6.0 \mathrm{~km} / \mathrm{s})$ & 9.5 \\
rendezvous & - & - & 26.7 & $0.24(7.3 \mathrm{~km} / \mathrm{s})$ & 11.4 \\
\hline
\end{tabular}

(a)

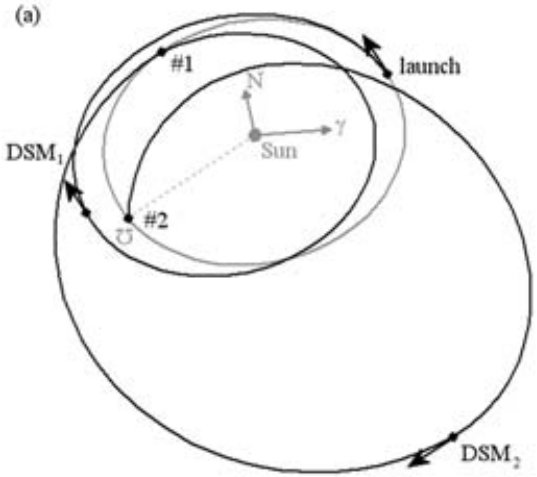

(b)

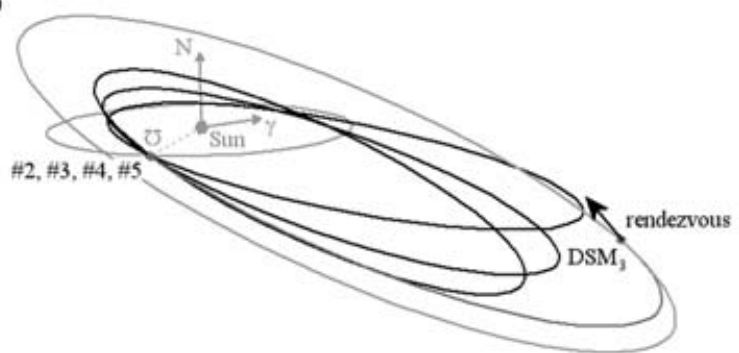

Figure 4. Schematic view of the transfer orbit found for the asteroid (1036) Ganymed. All velocity impulses are marked with arrows (the interplanetary insertion and three deep-space maneuvers). (a) Orbit of the Earth and powered transfer orbits used to increase the relative velocity of the spacecraft; (b) Orbit of the Earth, orbit of the asteroid, its true intersection orbit and resonant transfer orbits used to increase orbital inclination

on the target orbit. Second, the method was developed in the framework of a simplified theory of motion: piecewise two-body approximation. Because of that, it should be considered a first approximation transfer solution, which needs numerical improvement. Third, we did not take into account the phasing requirement between the spacecraft and the target asteroid. They should arrive (almost) simultaneously at the rendezvous point. But this is not hard to be accomplished, since the total travel time of the spacecraft can be slightly altered by changing some independent variables involved in computation or using another type of transfer orbit. Also, there is a launch opportunity every year, when the asteroid will have a different location on its orbit.

\section{References}

Battin, R. H. 1987, An introduction to the mathematics and methods of astrodynamics (AIAA Education Series)

Berinde, S. 2005 in: Z. Knežević \& A. Milani (eds.), Dynamics of Populations of Planetary Systems, Proceedings of the IAU Colloquium 197 (Cambridge University Press), p. 265

Carusi, A., Valsecchi, G. B. \& Greenberg, R. 1990, Celest. Mech. Dyn. Astron. 49, 111

Perozzi, E., Rossi, A. \& Valsecchi G. B. 2001, Planet. Space Sci. 49, 3 